TO APPEAR IN THE ASTROPHYSICAL JOURNAL

Preprint typeset using LTEX style emulateapj v. 5/2/11

\title{
SHORTEST RECURRENCE PERIODS OF NOVAE
}

\author{
MARIKO KATO \\ Department of Astronomy, Keio University, Hiyoshi, Yokohama 223-8521, Japan;
}

HIDEYUKI SAIO

Astronomical Institute, Graduate School of Science, Tohoku University, Sendai, 980-8578, Japan

IZUMI HACHISU

Department of Earth Science and Astronomy, College of Arts and Sciences, The University of Tokyo, 3-8-1 Komaba, Meguro-ku, Tokyo 153-8902, Japan AND

KEN'ICHI NOMOTO

Kavli Institute for the Physics and Mathematics of the Universe (WPI), The University of Tokyo, 5-1-5 Kashiwanoha, Kashiwa, Chiba 277-8583, Japan to appear in the Astrophysical Journal

\begin{abstract}
Stimulated by the recent discovery of the $1 \mathrm{yr}$ recurrence period nova M31N 2008-12a, we examined the shortest recurrence periods of hydrogen shell flashes on mass-accreting white dwarfs (WDs). We discuss the mechanism that yields a finite minimum recurrence period for a given WD mass. Calculating the unstable flashes for various WD masses and mass accretion rates, we identified a shortest recurrence period of about two months for a non-rotating $1.38 M_{\odot}$ WD with a mass accretion rate of $3.6 \times 10^{-7} M_{\odot} \mathrm{yr}^{-1}$. A $1 \mathrm{yr}$ recurrence period is realized for very massive $\left(\gtrsim 1.3 M_{\odot}\right)$ WDs with very high accretion rates $\left(\gtrsim 1.5 \times 10^{-7} M_{\odot} \mathrm{yr}^{-1}\right)$. We revised our stability limit of hydrogen shell burning, which will be useful for binary evolution calculations toward Type Ia supernovae.
\end{abstract}

Subject headings: nova, cataclysmic variables - stars: individual (M31N 2008-12a) - X-rays: binaries

\section{INTRODUCTION}

The recent discovery of the recurrent nova M31N 2008-12a has attracted attention to novae with short recurrence periods (Darnley et al. 2014; Henze et al. 2014; Tang et al. 2014). M31N 2008-12a showed the shortest recorded recurrence period of $1 \mathrm{yr}$, a very rapid turn-on of the stable supersoft X-ray source (SSS) phase, and a high effective temperature $(\sim 100 \mathrm{eV})$ in the SSS phase, all of which indicate a very massive white dwarf (WD). Such massive WDs in recurrent novae are considered to be one of the candidates for Type Ia supernova (SN Ia) progenitors (Hachisu et al. 1999a b; Hachisu \& Kato 2001; Hachisu et al. 2010; Han \& Podsiadlowski 2004; Li \& van den Heuvel 1997; Kato \& Hachisu 2012; Pagnotta \& Schaefer 2014). SNe Ia play very important roles in astrophysics as standard candles for measuring cosmological distances and as the main producers of iron group elements in the chemical evolution of galaxies. However, their immediate progenitors just before SN Ia explosions are still unclear. Thus, studies of novae with very short recurrence periods are essential to identify immediate progenitors of SNe Ia.

Many theoretical works on hydrogen shell flashes have been published. In general, short recurrence periods are obtained for very massive WDs with high mass accretion rates. When the mass accretion rate exceeds a certain value, nuclear burning is stable, and no shell flashes occur (Sienkiewics 1975, 1980; Sion et al. 1979; Iben 1982; Nomoto et al. 2007; Wolf et al. 2013). The border between stable and unstable mass accretion rates is known as the stability line, i.e., $\dot{M}_{\text {stable, }}$, in the diagram of accretion rate vs. WD mass. For a given

mariko@educ.cc.keio.ac.jp
WD mass, the shortest recurrence period is obtained near the stability line. However, it is not well known whether this minimum recurrence period approaches a finite value or zero. Wolf et al. (2013) recently obtained numerically the recurrence periods near the stability line for various WD masses and showed that the minimum recurrence period is finite. However, the theoretical reason for the finite value is still unclear. Moreover, the stability line obtained by Wolf et al. (2013) using a time evolution calculation differs slightly from that obtained using a linear stability analysis (Sienkiewics 1975, 1980; Nomoto et al. 2007). Because the stability line is important in binary evolution calculations toward SNe Ia, we examine the stability line of shell flashes and clarify why there is a finite minimum value of the recurrence period.

In the next section, we explain the reason for the finite minimum values of the nova recurrence period. In Section 3 , we present calculations of shell flashes on very massive WDs and numerically obtain the minimum recurrence periods for various WD masses. We also present a recalculated stability line, which could be useful for calculations of binary evolution. In Section 4 , we discuss some numerical calculations that resulted in shell flashes for mass accretion rates above the stability line. Finally, we summarize our results in Section 5.

\section{LIMIT CYCLE OF HYDROGEN SHELL FLASHES AND FINITE RECURRENCE PERIODS}

We first discuss the cycle of shell flashes using hydrostatic envelope models. We calculated the structures of the hydrogen-rich envelopes on a mass-accreting WD by solving the equations of hydrostatic equilibrium, mass continuity, energy conservation, and energy transfer together with the equation of state for degenerate matter (Eggleton et al 1973). We used the OPAL radiative opacities (Iglesias \& Rogers 1996). 


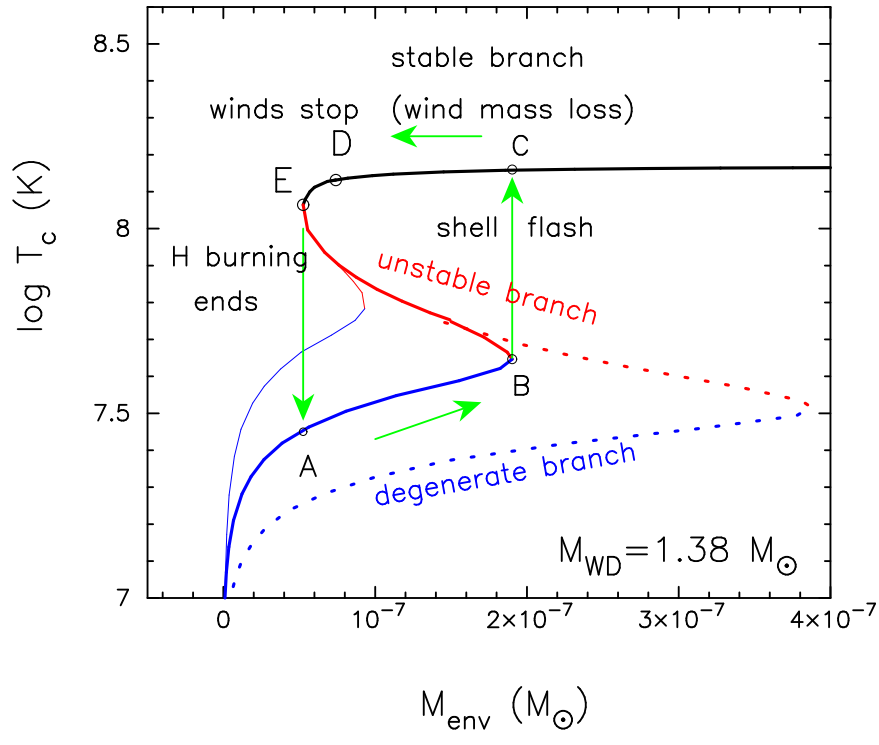

FIG. 1.- Schematic $M_{\mathrm{env}}-\log T_{\mathrm{c}}$ diagram for one cycle of nova outbursts on a $1.38 M_{\odot}$ WD with a given mass accretion rate $\dot{M}$. Here, $T_{\mathrm{c}}$ is the temperature at the bottom of the hydrogen-rich envelope, and $M_{\mathrm{env}}$ is the envelope mass. We plot three mass accretion rates: above the stability line, i.e., $\dot{M}>\dot{M}_{\text {stable }}$ (thin solid line); somewhat below the stability line, i.e., $\dot{M}<\dot{M}_{\text {stable }}$ (thick solid line); and much below the stability line, i.e., $\dot{M} \ll \dot{M}_{\text {stable }}$ (dotted line). For the thick solid line, the WD starts accreting around point $\mathrm{A}$ and the envelope mass increases. When it reaches turning point $\mathrm{B}$, a shell flash begins. Thus, the ignition mass is defined as $M_{\text {ig }}=M_{\text {env }}(\mathrm{B})$. Then the envelope expands and reaches point C. After an optical maximum at point $\mathrm{C}$, the envelope mass decreases owing to wind mass loss and nuclear burning. The optically thick wind stops at point D. Hydrogen burning ends at point $\mathrm{E}$, and the envelope quickly cools toward point $\mathrm{A}$. In this S-shaped sequence ABEDC, the lower branch (blue line from A to $\mathrm{B})$ represents a degenerate envelope, and the middle branch (red line from B to E) represents an unstable envelope for nuclear burning. The upper branch (black line from $\mathrm{E}$ to $\mathrm{C}$ ) represents an extended envelope after optical maximum, where nuclear burning is stable. For the lower mass accretion rate (dotted line), the degenerate branch is cooler owing to the smaller gravitational energy release; hence, the ignition mass is larger. For the higher mass accretion rate, the ignition mass is smaller. We found that the width of this limit cycle, $\Delta=M_{\mathrm{env}}(\mathrm{B})-M_{\mathrm{env}}(\mathrm{E})$, does not vanish even for very high mass accretion rates above the stability line (thin solid line). This is the reason for the minimum recurrence period of $t_{\mathrm{rec}}^{\min }=\Delta / \dot{M}_{\text {stable. }}$. See text for details.

The equation of energy conservation includes terms for nuclear burning, gravitational energy release, and radiative loss. For the gravitational energy release, we calculated the socalled homologous compressional heating, $\varepsilon_{g}^{\mathrm{h}}$, by accretion in a quasi-steady state on the $q$-coordinate $\left(q \equiv M_{r} / M\right)$, which is proportional to the mass accretion rate $\dot{M}$ (see, e.g., Neo et al. 1977; Kato 1980, 1982).

Figure 1 schematically depicts one cycle of shell flashes in the $M_{\mathrm{env}}-\log T_{\mathrm{c}}$ diagram for a $1.38 M_{\odot}$ WD with a given mass accretion rate of $\dot{M}$, where $M_{\text {env }}$ is the envelope mass, and $T_{\mathrm{c}}$ is the temperature at the bottom of the hydrogen-rich envelope. Here, we plot three mass accretion rates: above the stability line, i.e., $\dot{M}>\dot{M}_{\text {stable }}$ (thin solid line); somewhat below the stability line, i.e., $\dot{M}<\dot{M}_{\text {stable }}$ (thick solid line); and much below the stability line, i.e., $\dot{M} \ll \dot{M}_{\text {stable }}$ (dotted line). Note that the exact stability line will be determined by timedependent calculation of the shell flashes in Section 3.2. The hydrostatic solutions form an S-shaped sequence. The lowest branch, dubbed "the degenerate branch," represents a geometrically thin envelope in which most of the radiative energy loss is balanced by gravitational energy release because very little energy is generated by nuclear burning. On the mid- dle branch, dubbed "the unstable branch," the energy loss is balanced by the energy generated by hydrogen burning and gravitational energy release $\left(\varepsilon_{g}^{\mathrm{h}}\right)$. The energy generation by nuclear burning is comparable to or even dominates the gravitational energy release. Hydrogen shell burning on the middle branch is unstable. The upper branch, dubbed "the stable branch," represents an expanded phase of the nova outburst. On the right side to point $\mathrm{D}$, optically thick winds occur, and each envelope solution is constructed including wind mass loss (Kato \& Hachisu 1994). Hydrogen shell burning is stable on this branch (Kato 1983).

For the thick solid line $\left(\dot{M}<\dot{M}_{\text {stable }}\right)$ in Figure 1, a massaccreting WD begins its evolution from somewhere on the degenerate branch around point $\mathrm{A}$. The envelope mass increases with time because of accretion and moves rightward along the degenerate branch. After it reaches the turning point of the degenerate branch (point B), the hydrogen shell burning becomes unstable, triggering a shell flash. The envelope structure changes from a geometrically thin configuration to a very bloated one. The photosphere expands, and the photospheric temperature rapidly decreases. This is the onset of a nova outburst. The envelope mass at point B corresponds to the ignition mass, i.e., $M_{\mathrm{ig}}=M_{\mathrm{env}}(\mathrm{B})$.

After the WD jumps to point $\mathrm{C}$, it moves leftward as the envelope mass decreases owing to wind mass loss and nuclear burning (Kato \& Hachisu 1994; Kovetz 1998; Hachisu \& Kato 2001). This "stable branch" corresponds to expanded envelopes in the decay phase of a nova outburst. The photospheric radius gradually shrinks while the photospheric temperature increases. The optical brightness decays with time (Iben 1982; Kato \& Hachisu 1994; Hachisu \& Kato 2006). After the optically thick winds stop at point $\mathrm{D}$, the envelope mass continues to decrease owing to nuclear burning. As the envelope mass decreases, the radius of the envelope continues to decrease, so that the geometry of the envelope gradually changes from a spherically, extended configuration to a thin one. As a result, $T_{\mathrm{c}}$ decreases with the decreasing envelope mass from point $\mathrm{D}$ to $\mathrm{E}$. When the WD reaches a turning point (point $\mathrm{E}$ ), hydrogen burning becomes inactive because the envelope mass is too small to support high temperatures. The unstable branch starts at point E. The nuclear burning rate at point $E$ should be the same as that at the stability line, i.e.,

$$
\dot{M}_{\text {stable }}=\dot{M}_{\text {nuc }}(\mathrm{E})
$$

where $\dot{M}_{\text {nuc }}=L_{\mathrm{n}} / X Q$ is the nuclear burning rate, $L_{\mathrm{n}}$ is the luminosity of hydrogen burning, $X$ is the hydrogen mass fraction, and $Q=6.4 \times 10^{18} \mathrm{erg} \mathrm{g}^{-1}$ is the energy generation per unit mass. The star quickly falls to point $\mathrm{A}$ on the degenerate branch. In this way, a nova follows a limit cycle of points A, B, C, D, E, and then A.

Figure 1 also shows two other tracks for lower $\left(\dot{M} \ll \dot{M}_{\text {stable }}\right.$, dotted line $)$ and higher $\left(\dot{M}>\dot{M}_{\text {stable }}\right.$, thin solid line) mass accretion rates. For the lower mass accretion rate, the degenerate branch is cooler owing to the smaller gravitational energy release, but the position of the unstable branch (from point $\mathrm{E}$ to B) hardly changes. For this reason, point B is shifted rightward for the lower accretion rate; hence, the ignition mass is larger. For the higher mass accretion rate, on the other hand, point B moves leftward. As a result, the ignition mass is smaller. The higher the mass accretion rate is, the narrower the limit cycle of nova outbursts is.

The width of this limit cycle $\Delta$ is defined for each $\mathrm{S}$-shaped 
line as

$$
\Delta=M_{\mathrm{env}}(\mathrm{B})-M_{\mathrm{env}}(\mathrm{E}),
$$

where $M_{\mathrm{env}}(\mathrm{B})$ and $M_{\mathrm{env}}(\mathrm{E})$ are the envelope masses at turning points $\mathrm{B}$ and $\mathrm{E}$, respectively. We found that the width does not vanish even for very high mass accretion rates above the stability line $\left(\dot{M}>\dot{M}_{\text {stable, }}\right.$, thin solid line in Figure 1). In other words, the finite $\Delta$ is the reason for the finite minimum recurrence period of

$$
t_{\text {rec }}^{\min }=\Delta / \dot{M}_{\text {stable }},
$$

when the mass accretion rate increases toward the stability line, $\dot{M} \rightarrow \dot{M}_{\text {stable }}$.

Iben (1982) also presented the stability line in the HR diagram as a locus of minimum envelope masses of accreting WDs. The envelope mass has a minimum value at the knee near the highest temperature on the locus of an accreting WD. This corresponds to point E in Figure 1 Iben (1982) wrote that "the steady-burning solution is unstable along the low luminosity branch." Fuijimoto (1982) also discussed the stability change at point $\mathrm{E}$ in relation to the change of envelope structure from a spherical configuration (upper branch) to a flat configuration (lower branch). Sala \& Hernanz (2005) calculated the minimum envelope masses for various WD masses and chemical compositions ( $\mathrm{CO} / \mathrm{ONe}$ rich) using the OPAL opacities. They wrote, "Hydrogen burning will continue until the minimum envelope mass and maximum effective temperature are reached; evolution cannot proceed further with stationary hydrogen-burning." Thus, hydrogen burning is extinguished, and the envelope enters the cooling phase.

The tracks in Figure 1 differ slightly from those of our timedependent calculations, as will be described in detail in Section 3 . We will identify the exact stability line using a timedependent calculation in Section 3.2

Finally, we discuss the case that the mass accretion rate is larger than that of the stability line. For the thin solid line $\left(\dot{M}>\dot{M}_{\text {stable }}\right)$ in Figure 1, the WD accretes hydrogen-rich matter and reaches turning point $\mathrm{B}$. Hydrogen ignites to trigger a shell flash. After the WD jumps from turning point B to point $\mathrm{C}$, it stays at a point on the stable branch to the right of point $\mathrm{E}$ because the mass accretion rate is larger than the nuclear burning rate at point E; i.e., $\dot{M}>\dot{M}_{\text {nuc }}(\mathrm{E})=\dot{M}_{\text {stable }}$. We emphasize that, after the first shell flash occurs, the WD stays at a point on the stable branch, and the shell flashes never repeat. We will discuss this in more detail in Section 4.2

\section{SIMULATION OF SHELL FLASHES}

In this section, we present and discuss the results of time evolution calculations for part of the nova cycle. We used a Henyey-type evolution code, which is the same as that in Nomoto et al. (2007) except that a term proportional to $(\partial s / \partial t)_{q}$, where $s$ is the specific entropy, is included to follow the evolution. The chemical composition of the accreting matter and initial hydrogen-rich envelope is assumed to be $X=0.7, Y=0.28$, and $Z=0.02$, except for the cases in section 4.3 Neither convective overshooting nor diffusion processes are included; thus, no WD material is mixed into the hydrogen-rich envelope.

As the initial model, we adopted an equilibrium model (i.e., "the steady-state models" of Nomoto et al. 2007), in which an energy balance is already established between heating by mass accretion and nuclear energy generation and cooling by radiative transfer and neutrino energy loss. This is a good

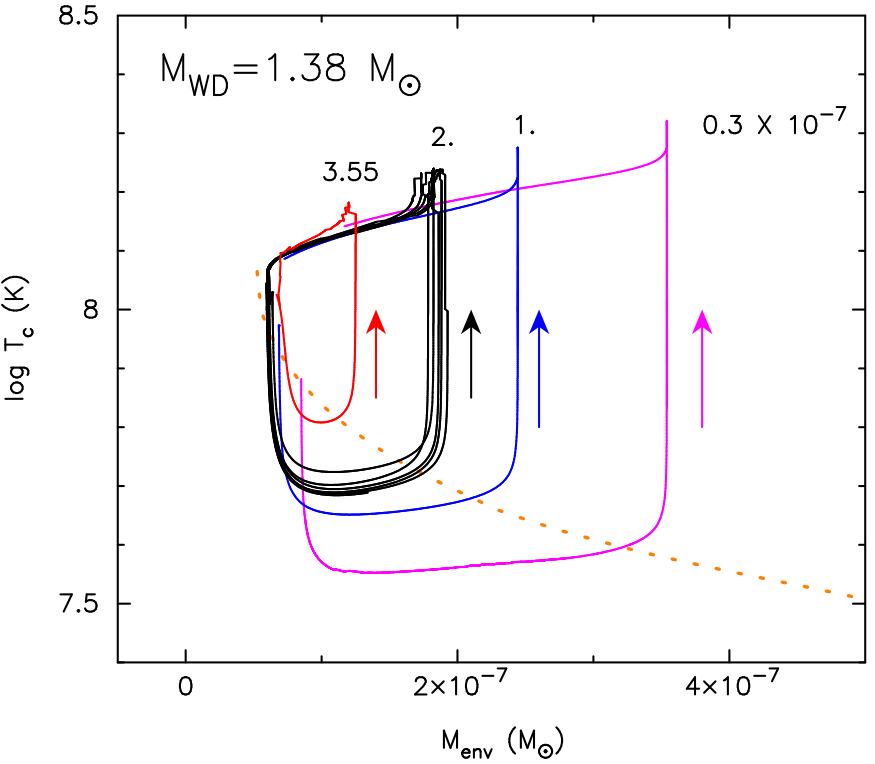

FIG. 2.- Same as Figure 1 but for time-dependent calculation on a $1.38 M_{\odot}$ WD. From left to right, the mass accretion rate is $\dot{M}=3.55 \times 10^{-7}$ (red solid line), $2 \times 10^{-7}$ (black solid line), $1 \times 10^{-7}$ (blue solid line), and $0.3 \times 10^{-7} M_{\odot} \mathrm{yr}^{-1}$ (magenta solid line), as indicated next to each curve in units of $10^{-7} M_{\odot} \mathrm{yr}^{-1}$. For $2 \times 10^{-7} M_{\odot} \mathrm{yr}^{-1}$, five cycles are shown, whereas for the other cases, the first cycle is shown. We define the ignition mass $M_{\text {ig }}=M_{\text {env }}$ as the envelope mass at each vertical line of the first shell flash. An orange dotted line shows the unstable branch in Figure 1

approximation, as we will see later, of the long time-averaged evolution of a mass accreting WD. Thus, starting from such an initial equilibrium state, we expect that the ignition mass estimated on the basis of the first flash is close to the final one.

Using this equilibrium model for the initial evolution model is advantageous because, after many flashes, the temperature distribution in the WD interior in a recurrent nova is expected to be similar to that of the equilibrium model accreting at the same rate. This is a good approximation, in particular, for short-period recurrent novae with very high accretion rates. We assumed no helium layer between the WD core and hydrogen-rich envelope. In some cases, we followed several shell flashes to confirm that the equilibrium approximation is adequate.

Henyey-type evolution codes are well known to have difficulties in calculating extended envelopes after a shell flash. To continue our calculation, we assumed large mass loss rates so that the radius does not exceed $0.01-0.02 R_{\odot}$. The mass loss rate thus determined is artificial and does not represent any physical processes in nova outbursts. Although we adopted the smallest possible mass loss rates, this artificial mass loss would shorten the duration of a flash (i.e., the high state). We suppose, however, that it has little effect on the resultant recurrence period because the durations of the flashes are much shorter than the recurrence period.

\subsection{Ignition Mass}

We obtain the ignition mass for a given WD mass and mass accretion rate as follows: We start our evolution calculation by reducing the envelope mass to half that of the corresponding equilibrium model. The nuclear luminosity $L_{\mathrm{n}}$ initially decreases because of the reduced envelope mass. This process corresponds to a falling path from a point on the unstable equilibrium branch toward the degenerate branch in Figure 11. As the envelope mass is increased by accretion, the 


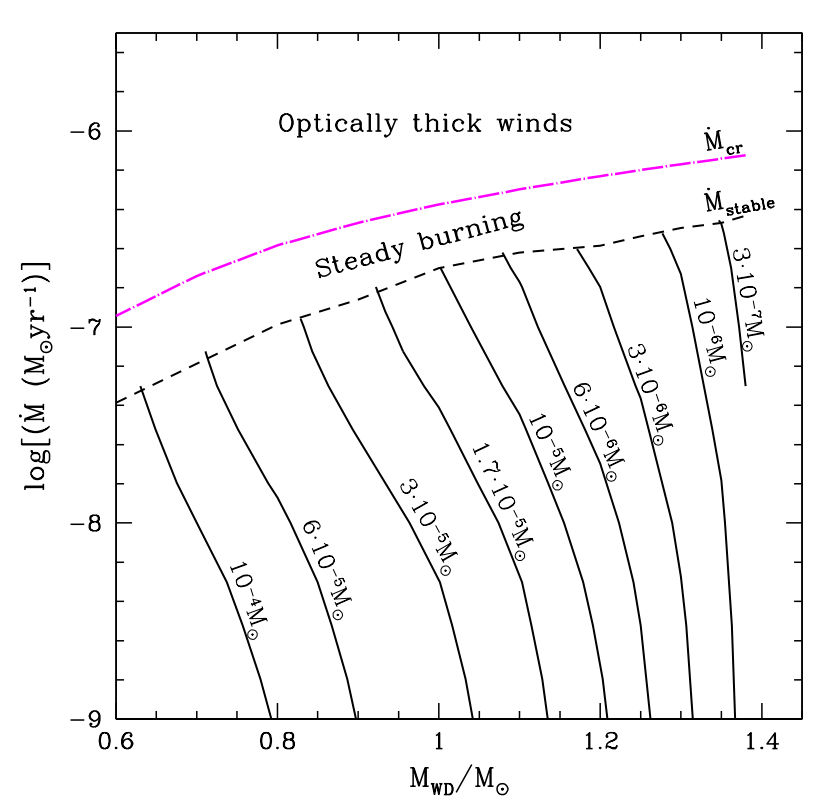

FIG. 3.- Ignition mass $M_{\mathrm{ig}}$ on the $M_{\mathrm{WD}}-\dot{M}$ plane. Hydrogen burning is stable in the region above the dashed line $\left(\dot{M}_{\text {stable }}\right)$. In the region below $\dot{M}_{\text {stable }}$, hydrogen shell burning is thermally unstable, and the WD experiences shell flashes. Black solid lines indicate equi-ignition masses, the values of which are shown beside each line. Optically thick winds are accelerated in the region above the dash-dotted line $\left(\dot{M}_{\mathrm{cr}}\right)$.

WD moves rightward and reaches point $\mathrm{B}$, at which hydrogen burning becomes unstable, and a shell flash occurs. We define the ignition mass $M_{\mathrm{ig}}=M_{\mathrm{env}}$ as the envelope mass at the onset of the flash. The ignition mass obtained in this way depends very little on the process or amount of initial reduction of the envelope mass; e.g., no reduction or a $90 \%$ reduction yields a similar value of $M_{\text {ig }}$.

Figure 2 shows the evolutionary courses of these calculations for a $1.38 M_{\odot}$ WD in the $M_{\text {env }}-\log T_{\mathrm{c}}$ diagram for various mass accretion rates. The orange dashed line represents the unstable branch in Figure 1. After a steep decrease in $T_{\mathrm{c}}$ (and hence in the nuclear luminosity $L_{\mathrm{n}}$ ), the envelope mass $M_{\text {env }}$ increases to the ignition mass $M_{\text {ig }}$, and a shell flash starts. As the shell flash sets in, the temperature $T_{\mathrm{c}}$ and nuclear energy generation rate $L_{\mathrm{n}}$ increase rapidly, so the entire envelope expands. We define the ignition mass as the mass at which the temperature rises steeply in the first flash. Although the ignition mass changes slightly for every flash, as shown for the $2 \times 10^{-7} M_{\odot} \mathrm{yr}^{-1}$ case, the ignition mass at the first flash is sufficiently close to that of the limit cycle. This is one of the merits of starting from the equilibrium model. The ignition mass thus determined will be examined in more detail in Section 4.4

We obtained the ignition masses $M_{\mathrm{ig}}$ for various sets of mass accretion rates $\dot{M}$ and WD masses $M_{\mathrm{WD}}$. The results are presented as contours of $M_{\mathrm{ig}}$ on the $M_{\mathrm{WD}}-\dot{M}$ plane in Figure 3 For a given $\dot{M}, M_{\mathrm{ig}}$ is smaller for a larger $M_{\mathrm{WD}}$. For a given $M_{\mathrm{WD}}, M_{\mathrm{ig}}$ is smaller for a higher $\dot{M}$. All the contours stop at the dashed line because above this line, hydrogen shell burning is stable, and no flashes occur; in other words, it is the stability line for hydrogen shell burning. The stability line

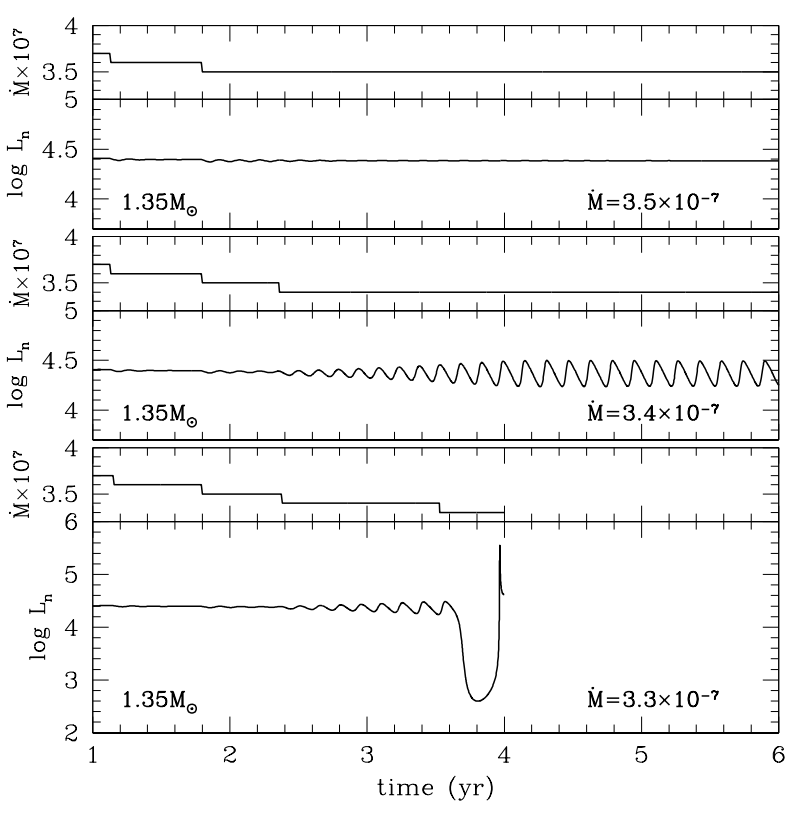

FIG. 4.- Evolutions of nuclear luminosity $L_{\mathrm{n}}$ (in units of $L_{\odot}$ ) for selected accretion rates $\dot{M}$ (in units of $M_{\odot} \mathrm{yr}^{-1}$ ) around the boundary between stable and unstable shell hydrogen burning for a $1.35 M_{\odot}$ WD. Each calculation started with the equilibrium model for an accretion rate of $4.5 \times 10^{-7} M_{\odot} \mathrm{yr}^{-1}$, which is high enough for stable hydrogen shell burning. The accretion rate is gradually decreased (upper sub-panel of each panel) to $\dot{M}=3.5 \times 10^{-7} M_{\odot} \mathrm{yr}^{-1}$ in the top panel, $\dot{M}=3.4 \times 10^{-7} M_{\odot} \mathrm{yr}^{-1}$ in the middle panel, and $\dot{M}=3.3 \times 10^{-7} M_{\odot} \mathrm{yr}^{-1}$ in the bottom panel. The stability of the shell burning changes at $\dot{M}=3.4 \times 10^{-7} M_{\odot} \mathrm{yr}^{-1}$ (middle panel), at which the nuclear luminosity oscillates without leading to a flash. For an accretion rate slightly smaller than the above value, a shell flash occurs (bottom panel).

(dashed line in Figure 3) is approximately represented by

$$
\dot{M}_{\text {stable }}=4.17 \times 10^{-7}\left(\frac{M_{\mathrm{WD}}}{M_{\odot}}-0.53\right) M_{\odot} \mathrm{yr}^{-1} .
$$

We will discuss how the stability line is obtained in the next subsection.

The dash-dotted line in Figure 3 represents the locus of the critical accretion rate $\dot{M}_{\mathrm{cr}}$, above which steady models have optically thick winds (Kato \& Hachisu 1994). This relation can be represented as

$$
\dot{M}_{\mathrm{cr}}=8.18 \times 10^{-7}\left(\frac{M_{\mathrm{WD}}}{M_{\odot}}-0.48\right) M_{\odot} \mathrm{yr}^{-1} .
$$

\subsection{Stability Line}

To obtain the stability boundary of the accretion rate, we start the evolution calculations from an equilibrium model whose accretion rate lies well within the stable range. Then, we decrease the accretion rate slowly until a shell flash occurs. Figure 4 depicts examples for $M_{\mathrm{WD}}=1.35 M_{\odot}$. The initial model has a mass accretion rate of $\dot{M}=4.5 \times 10^{-7} M_{\odot} \mathrm{yr}^{-1}$. In the top panel, the accretion rate $\dot{M}$ is reduced to $3.5 \times$ $10^{-7} M_{\odot} \mathrm{yr}^{-1}$. The hydrogen shell burning is still stable at this accretion rate, and the nuclear luminosity $L_{\mathrm{n}}$ remains nearly constant.

In the middle panel, $\dot{M}$ is decreased to $3.4 \times 10^{-7} M_{\odot} \mathrm{yr}^{-1}$. The nuclear luminosity $L_{\mathrm{n}}$ oscillates, but the amplitude is 


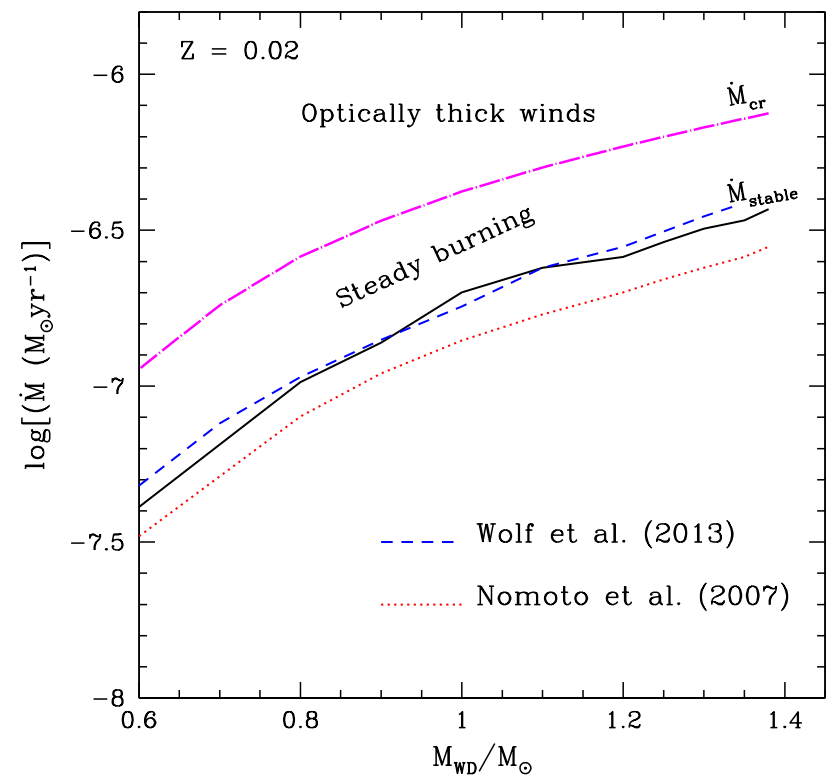

FIG. 5.- Comparison of stability line calculated in this work (black solid line) with those of Nomoto et al. (2007) (red dotted line) and Wolf et al. (2013) (blue dashed line) in the $M_{\mathrm{WD}}-\dot{M}$ diagram. Other symbols/lines are the same as in Figure 3 See Section 3.2 for details.

bounded. If $\dot{M}$ is further decreased to $3.3 \times 10^{-7} M_{\odot} \mathrm{yr}^{-1}$, the nuclear luminosity $L_{\mathrm{n}}$ starts decreasing, and after a certain mass is accreted, a shell flash occurs (bottom panel). The stability of hydrogen shell burning changes at $\dot{M}=3.4 \times$ $10^{-7} M_{\odot} \mathrm{yr}^{-1}$ for $M_{\mathrm{WD}}=1.35 M_{\odot}$. We regard this accretion rate as the boundary of stability.

We note that the appearance of oscillations in $L_{\mathrm{n}}$ for the models with accretion rates around the stability limit is consistent with the prediction made by Paczyński (1983) from a linear stability analysis for a simplified plane-parallel onezone model. He found that the eigenvalues of the linear analysis are always complex near the transition from stability to instability because of the similarity between the thermal and nuclear timescales, which means that the temporal variations in the perturbations are oscillatory.

Figure 5 compares the stability line we obtained (black solid line) with those of Nomoto et al. (2007) (red dotted line) and Wolf et al. (2013) (blue dashed line). Nomoto et al. (2007) obtained the stability line from a linear perturbation analysis of equilibrium models, whereas Wolf et al. (2013) obtained it based on their evolution calculations. The two lines based on time evolution calculations agree well with each other, whereas that based on the linear perturbation analysis lies below them.

The reason for the difference is not clear. Although the linear stability analysis of Nomoto et al. (2007) includes only thermal effects, and hence only real eigenvalues are expected, Paczyński (1983) showed that the monotonic thermal stability transition occurs at the same accretion rate as oscillatory instability. The difference might stem from small differences in the structures between the equilibrium models and time evolution models.

\subsection{Shortest Recurrence Periods}

Now that we have obtained $M_{\mathrm{ig}}$ for various $M_{\mathrm{WD}}$ and $\dot{M}$, we can approximate the recurrence periods of novae. After a nova outburst sets in, the envelope mass of the WD decreases as its surface temperature increases. Hydrogen burning stops at point $\mathrm{E}$ in Figure 1 when the envelope mass reaches a local minimum, $M_{\mathrm{env}}^{\mathrm{min}}$. This hydrogen-rich matter remains unburnt until the next flash occurs; during this time, an amount of mass equal to $M_{\mathrm{ig}}-M_{\mathrm{env}}^{\mathrm{min}}$ is accreted. We estimate the recurrence period as $t_{\text {rec }}=\left(M_{\mathrm{ig}}-M_{\mathrm{env}}^{\mathrm{min}}\right) / \dot{M}$ because the time after ignition to point $\mathrm{E}$ is much shorter than the accretion time. We show in Section 4.4 that the recurrence periods determined in this way are consistent with the evolution calculations of several flashes.

Figure 6 shows the contours of the recurrence period on the $M_{\mathrm{WD}}-\dot{M}$ plane. The recurrence period of $1 \mathrm{yr}$ corresponding to the nova M31N 2008-12a is obtained for WDs with $\left(\dot{M}, M_{\mathrm{WD}}\right)=\left(3.3 \times 10^{-7} M_{\odot} \mathrm{yr}^{-1}, 1.31 M_{\odot}\right),(2.4 \times$ $\left.10^{-7} M_{\odot} \mathrm{yr}^{-1}, 1.35 M_{\odot}\right)$, and $\left(1.5 \times 10^{-7} M_{\odot} \mathrm{yr}^{-1}, 1.38 M_{\odot}\right)$. In multicycle nova outbursts calculated by Prialnik \& Kovetz (1995), a 1 yr recurrence period corresponds to massive WDs such as $\left(\dot{M}, M_{\mathrm{WD}}\right)=\left(\sim 10^{-7} M_{\odot} \mathrm{yr}^{-1}, 1.4 M_{\odot}\right)$. Wolf et al. (2013) and Tang et al. (2014) also obtained a $1 \mathrm{yr}$ period for $\left(\dot{M}, M_{\mathrm{WD}}\right)=\left(3.4 \times 10^{-7} M_{\odot} \mathrm{yr}^{-1}, 1.30 M_{\odot}\right)$ and $\left(1.7 \times 10^{-7} M_{\odot} \mathrm{yr}^{-1}, 1.36 M_{\odot}\right)$. Our values are consistent with these works.

For a given $\dot{M}$, the recurrence period is shorter for a larger $M_{\mathrm{WD}}$. For a given $M_{\mathrm{WD}}$, the recurrence period is shorter for a higher $\dot{M}$. The latter is limited by the stability line, as indicated by the dashed line in Figure 6, which gives a lower bound of the recurrence period for a given $M_{\mathrm{WD}}$. We should note that for the accretion rates as high as $\dot{M}>10^{-7} M_{\odot} \mathrm{yr}^{-1}$ encountered here, the accreting $\mathrm{C}+\mathrm{O}$ WD becomes an $\mathrm{SN}$ Ia when $M_{\mathrm{WD}}$ reaches $1.38 M_{\odot}$ because carbon deflagration is ignited at the center of the WD (Nomoto 1982; Nomoto et al. 1984; Hillebrandt \& Niemeyer 2000). If the accreting WD is an $\mathrm{O}+\mathrm{Ne}+\mathrm{Mg} \mathrm{WD}$, electron capture triggers a collapse when $M_{\mathrm{WD}}=1.38 M_{\odot}($ Nomoto 1984; Canal et al. 1990). Therefore, the shortest recurrence period of novae is obtained for the $1.38 M_{\odot}$ WD with an accretion rate of $3.6 \times 10^{-7} M_{\odot} \mathrm{yr}^{-1}$, and is found to be about 2 months (see Figure 8 below).

\section{DISCUSSION}

\subsection{Comparison with Other Works: Under the Stability Line}

Prialnik \& Kovetz (1995) calculated the shell flashes for various WD masses and accretion rates. For $\dot{M}=1 \times$ $10^{-7} M_{\odot} \mathrm{yr}^{-1}$ with a core temperature of $T_{\mathrm{c}}=5 \times 10^{7} \mathrm{~K}$, they obtained the WD mass, accreted mass, and recurrence period as $\left(1.0 M_{\odot}, 8.30 \times 10^{-6} M_{\odot}, 83 \mathrm{yr}\right),\left(1.25 M_{\odot}, 1.96 \times\right.$ $\left.10^{-6} M_{\odot}, 19.6 \mathrm{yr}\right)$, and $\left(1.4 M_{\odot}, 8.09 \times 10^{-8} M_{\odot}, 0.809 \mathrm{yr}\right)$. For the same accretion rate of $1 \times 10^{-7} M_{\odot} \mathrm{yr}^{-1}$, Idan et al. (2013) reported values of $\left(1.0 M_{\odot}, 9 \times 10^{-6} M_{\odot}, 100 \mathrm{yr}\right)$, $\left(1.25 M_{\odot}, 1.5 \times 10^{-6} M_{\odot}, 16\right.$ yr $),\left(1.35 M_{\odot}, 3.5 \times 10^{-7} M_{\odot}\right.$, $3.7 \mathrm{yr})$, and $\left(1.4 M_{\odot}, 5 \times 10^{-8} M_{\odot}, 0.5 \mathrm{yr}\right)$. Our models yield $\left(1.0 M_{\odot}, 1.3 \times 10^{-5} M_{\odot}, 95 \mathrm{yr}\right),\left(1.25 M_{\odot}, 2.3 \times 10^{-6} M_{\odot}\right.$, $17 \mathrm{yr}),\left(1.35 M_{\odot}, 5.5 \times 10^{-7} M_{\odot}, 4.2 \mathrm{yr}\right)$, and $\left(1.38 M_{\odot}\right.$, $\left.2.4 \times 10^{-7} M_{\odot}, 1.8 \mathrm{yr}\right)$. These values are more or less consistent with each other. In our model, the WD is heated by gravitational energy release due to high mass accretion rates. As a result, the WD radius (i.e., the bottom of the envelope) should be slightly larger than those of Idan et al. (2013) and Prialnik \& Kovetz (1995). Thus, our envelope mass is larger than theirs because of the smaller surface gravity of the WD.

Near the stability line, our ignition mass $M_{\mathrm{ig}}$ is very con- 


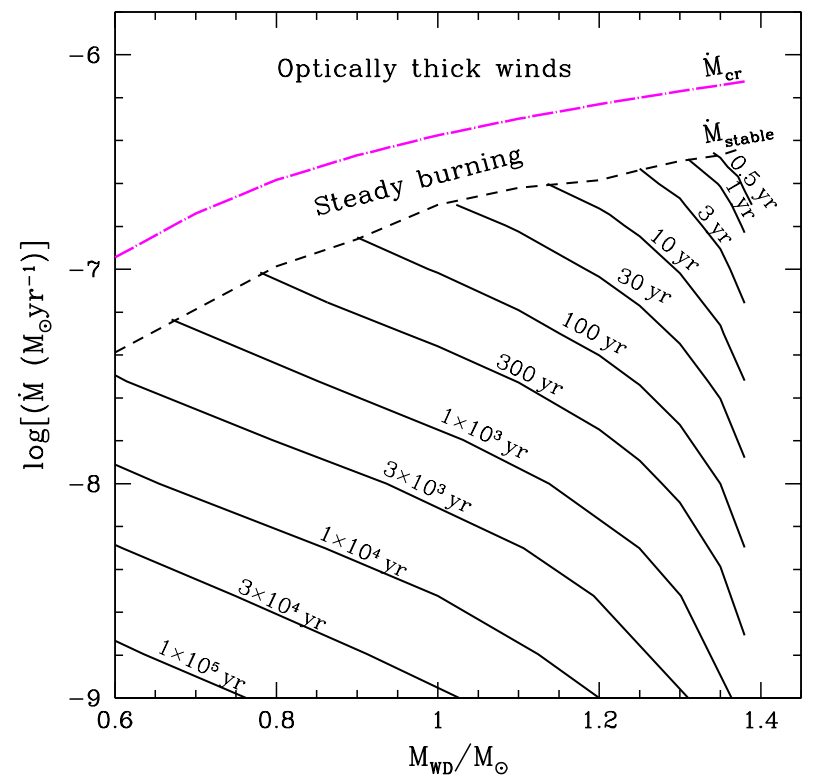

FIG. 6.- Recurrence period $t_{\text {rec }}$ of novae on the $M_{\mathrm{WD}}-\dot{M}$ plane. We plot the loci of the equi-recurrence periods of novae (black solid lines). Other symbols/lines are the same as in Figure 3

sistent with the results of Wolf et al. (2013), as indicated by the agreement of the two stability lines in Figure 5] For high mass accretion rates, Wolf et al.'s models develop a hot helium layer below the accreted hydrogen-rich envelope after the WD experiences several shell flashes. Wolf et al. claimed that the presence of a hot helium layer is essential for nova outbursts of short recurrence periods because it acts as a heat reservoir even if the WD is cool. Our equilibrium model has no helium layer but has a hot WD core, which is heated by compressional heating due to high mass accretion rates. For example, the maximum temperature of our $1.0 M_{\odot}$ WD model with $\dot{M}=1.0 \times 10^{-7} M_{\odot} \mathrm{yr}^{-1}$ reaches $\log T(\mathrm{~K})=8.07$ at the outer part of the WD core, which is comparable with that of Wolf et al.'s helium layer. Therefore, our models and Wolf et al.'s have a similar inner boundary condition in view of the heat reservoir at the bottom of the hydrogen-rich envelope, and thus give very consistent results.

\subsection{Shell Flashes above the Stability Line: Artifact Flashes}

Although the stability line of shell flashes in Figure 3 has long been established (Iben 1982; Nomoto 1982; Nomoto et al. 2007; Paczyński \& Żytkow 1978; Sienkiewics 1975, 1980; Sion et al. 1979; Wolf et al. 2013), some numerical calculations showed repeated shell flashes for accretion rates even above the stability line (Kovetz \& Prialnik 1994; Prialnik \& Kovetz 1995; Starrfield et al. 2012; Idan et al. 2013). In these cases, the recurrence period is much shorter than our minimum value, and the light curves show a very high duty cycle. For example, Kovetz \& Prialnik (1994) reported the calculation of several successive shell flashes on a $1.4 M_{\odot}$ WD with $\dot{M}=$ $1 \times 10^{-6} M_{\odot} \mathrm{yr}^{-1}$. The bolometric luminosity varies between $\log \left(L_{\mathrm{bol}} / L_{\odot}\right) \sim 3.7$ and $\sim 4.8$, and the bright stage lasts 10 days, almost half of the recurrence period of 20 days. Idan et al. (2013) obtained successive nova outbursts for a $1.0 M_{\odot}$ WD with $\dot{M}=1 \times 10^{-6} M_{\odot} \mathrm{yr}^{-1}$. The bright stage lasts

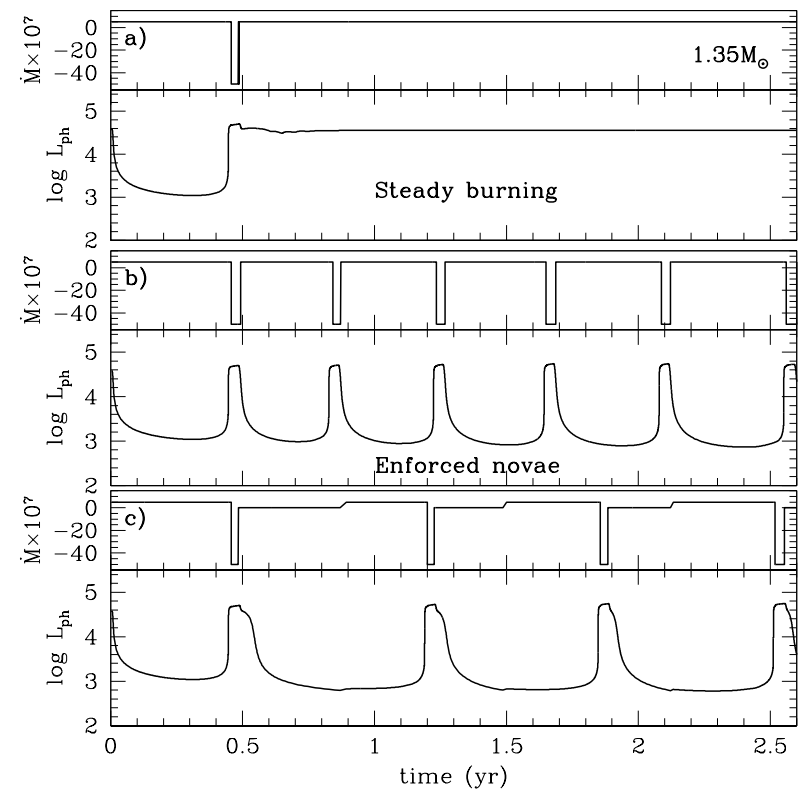

FIG. 7.- Evolutions of photospheric luminosity $\log L_{\mathrm{ph}}$ (in units of $L_{\odot}$ ) of a $1.35 M_{\odot}$ WD for an accretion rate of $\dot{M}=5 \times 10^{-7} M_{\odot} \mathrm{yr}^{-1}$, above the stability line. Each calculation started with about half of the equilibrium envelope mass; therefore, the envelope underwent a first shell flash at about 0.45 yr. To avoid numerical difficulty, we assumed an artificial wind mass loss of $\dot{M}=-5 \times 10^{-6} M_{\odot} \mathrm{yr}^{-1}$ when the photosphere expands to $\log R_{\mathrm{ph}} / R_{\odot}>$ -1.7 . In the upper part of each panel, we plot our control mass accretion/mass loss rate in units of $10^{-7} M_{\odot} \mathrm{yr}^{-1}$. (a) Upper panel: after the first shell flash, we stopped the wind mass loss and restarted mass accretion when the photospheric radius shrank to $\log R_{\mathrm{ph}} / R_{\odot}=-2.0$, where the envelope had not yet reached point $\mathrm{E}$ in Figure 1 Hydrogen burning was occurring (steady state). The envelope remained on the stable branch in Figure 1 (b) Middle panel: after the first shell flash, we stopped the wind mass loss and restarted mass accretion when the photospheric radius shrank to $\log R_{\mathrm{ph}} / R_{\odot}=-2.4$, where the envelope had already reached point $\mathrm{E}$ in Figure 1 and began to fall toward point A. The envelope repeated a limit cycle (enforced novae). (c) Bottom panel: after the first shell flash, we stopped the wind mass loss when the photospheric radius shrank to $\log R_{\mathrm{ph}} / R_{\odot}=-2.0$, but did not restart mass accretion, i.e., $\dot{M}=0$, until the photospheric luminosity decreases to $\log L_{\mathrm{ph}} / L_{\odot}<2.8$. The envelope gradually moved toward and reached point $\mathrm{E}$ in Figure 11 After the envelope fell toward point A, we restarted mass accretion. The envelope again underwent a shell flash (enforced nova). The recurrence period is longer than in the middle panel.

$6 \mathrm{yr}, 60 \%$ of the total recurrence period of $10 \mathrm{yr}$. Both of these calculations show a long lasting high-luminosity phase during the relatively short recurrence period. Such light curves do not resemble those of recurrent novae, which show much shorter duty cycles.

The accretion rate of $\dot{M}=1 \times 10^{-6} M_{\odot} \mathrm{yr}^{-1}$ corresponds to the region above the stability line in Figure 3, in which hydrogen shell burning should be stable, as discussed in Section 2 (Figure 11). Nevertheless, these papers reported successive nova outbursts. This is because they stopped accretion during the shell flashes. Without a supply of hydrogen (nuclear fuel), a nova evolves toward point E in Figure 1, where it immediately falls to point A. These authors then restarted accretion. After some time, the next shell flash begins, and it goes up from point B to point C. In this way, these authors switched mass accretion on and off to run the limit cycle. In other words, these outbursts are "enforced novae," and it is not known whether such an on/off switch works in nova outbursts or in other accreting binaries.

Figure 7 shows examples of our numerical simulations 
for such enforced novae for a $1.35 M_{\odot}$ WD with $\dot{M}=5 \times$ $10^{-7} M_{\odot} \mathrm{yr}^{-1}$, which is above the stability line. We show three cases for different on/off switches for mass accretion. Each calculation started with about half of the equilibrium envelope mass; therefore, the envelope underwent the first shell flash at about $0.45 \mathrm{yr}$. To avoid numerical difficulty, we assumed an artificial wind mass loss of $\dot{M}=-5 \times 10^{-6} M_{\odot} \mathrm{yr}^{-1}$ when the photosphere expands greatly $\left(\log R_{\mathrm{ph}} / R_{\odot}>-1.7\right)$. (a) Upper panel: after the first shell flash, we stopped the wind mass loss when the photospheric radius shrank to $\log R_{\mathrm{ph}} / R_{\odot}=-2.0$, where the envelope did not yet reach point $\mathrm{E}$ in Figure 1 , and immediately restarted mass accretion. Hydrogen burning was occurring (steady state). The envelope remained on the stable branch in Figure 1 In the upper part of each panel, we plot our control mass accretion/mass loss rate in units of $10^{-7} M_{\odot} \mathrm{yr}^{-1}$. (b) Middle panel: after the first shell flash, we stopped the wind mass loss and immediately restarted mass accretion when the photospheric radius shrank to $\log R_{\mathrm{ph}} / R_{\odot}=-2.4$, which is a bit later than in (a). The envelope had already passed through point $\mathrm{E}$ in Figure 1 and fell toward point A. After we restarted mass accretion, the envelope repeated a limit cycle. This is the enforced nova outburst. (c) Bottom panel: after the first shell flash, we stopped the wind mass loss when the photospheric radius shrank to $\log R_{\mathrm{ph}} / R_{\odot}=-2.0$, the same time as in (a), but did not restart mass accretion, i.e., $\dot{M}=0$, until the photospheric luminosity further decreased to $\log L_{\mathrm{ph}} / L_{\odot}<2.8$. We restarted mass accretion after the envelope fell from point $E$ toward point $A$ in Figure 1. The envelope again underwent a shell flash (enforced nova). The recurrence period was longer than in the middle panel because we delayed the restart of mass accretion. In this way, we can control the recurrence period by changing the time at which mass accretion is restarted. The above three cases clearly show that shell flashes above the stability line are just artifacts that stem from the numerical treatment of the mass accretion on/off switch.

There is some indication that accretion does not stop in real binaries. When the accretion rate is between the two lines of $\dot{M}_{\text {cr }}$ and $\dot{M}_{\text {stable }}$ in Figure 3 , the WD burns hydrogen at the same rate as the accretion rate and stays somewhere between points D and E in Figure 1 These binaries correspond to steady SSSs such as CAL 83, 1E0035.4-7230, and RX J0925.7-4758 (e.g., van den Heuvel et al. 1992; Kahabka \& van den Heuvel 1997; Nomoto et al. 2007). In these binaries, the WD is considered to accrete matter from the disk and to emit X-rays from the other WD surface area.

If the accretion rate is larger than $\dot{M}_{\mathrm{cr}}$, the star stays somewhere to the right of point D in Figure 1, where optically thick winds occur. The mass accretion rate is balanced with the sum of the nuclear burning rate and the wind mass loss rate. In this case, the WD accretes matter from the disk and ejects excess matter in the wind from the other regions. RX J0513.9-6951 and V Sge are considered to be such objects (Hachisu \& Kato 2003a b).

\subsection{Effects of Chemical Composition}

The ignition mass depends on the chemical composition of the envelope as well as the WD mass and mass accretion rate. Figure 8 compares the ignition mass and recurrence period for $X=0.1, Y=0.88$, and $Z=0.02$ with those for the solar composition. The ignition mass for $X=0.1$ is systematically larger than that for $X=0.7$. The nuclear burning rate due to the $p p$ chain is proportional to $X^{2}$, and the rate of the CNO cycle is

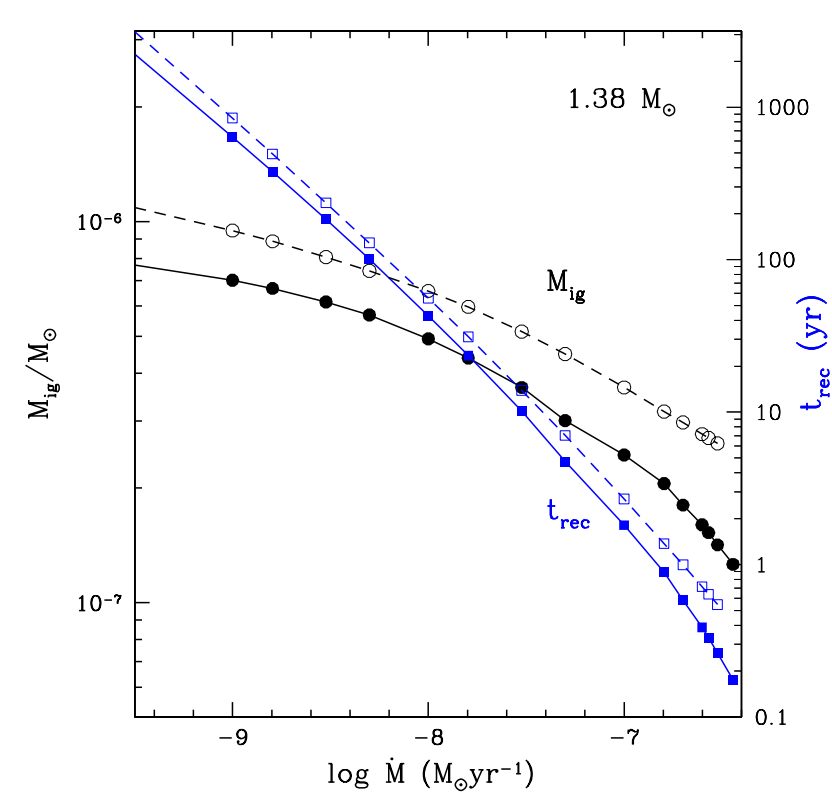

FIG. 8.- Recurrence period and ignition mass of novae on a $1.38 M M_{\odot}$ WD. Black lines/symbols represent ignition masses (left vertical axis). Blue lines/symbols represent recurrence period $t_{\mathrm{rec}}$ (right vertical axis). Solid lines with filled symbols indicate the case of $X=0.7, Y=0.28$, and $Z=0.02$, whereas dashed lines with open symbols denote the case of $X=0.1, Y=$ 0.88 , and $Z=0.02$. The minimum recurrence period is about two months for $X=0.7, Y=0.28$, and $Z=0.02$

proportional to $X$. For smaller $X$, unstable nuclear burning ignites at a higher temperature $T_{\mathrm{c}}$, which results in a more massive envelope. This will be useful in binary evolution calculations toward SNe Ia, in which a WD accretes He-rich matter, as suggested for U Sco type binaries (Hachisu et al. 1999a).

\subsection{Recurrence Periods after Multicycle Nova Outbursts}

The ignition mass shown in Figure 3 is obtained from the first shell flash of the model evolved from the equilibrium model. The recurrence time of nova outbursts in Figure 6 is estimated from the ignition mass and the minimum envelope mass for each WD mass. To verify these estimates, we calculated several consecutive shell flashes starting from the equilibrium model for selected cases.

Figure 2 shows five successive cycles of shell flashes for $M_{\mathrm{WD}}=1.38 M_{\odot}$ and $\dot{M}=2 \times 10^{-7} M_{\odot} \mathrm{yr}^{-1}$. These five tracks are very similar. In general, if we start from an arbitrary initial thermal condition of the WD, for example, a cold WD, the strength and recurrence period of the shell flashes would vary from flash to flash and gradually approach a final limit cycle (see e.g. Iben 1982; Kovetz \& Prialnik 1994). To avoid a lengthy calculation, as the initial model we adopted the equilibrium model, which is a good approximation of the long time-averaged evolution of WDs, as shown in Figure 2 and also the discussion below.

Figure 9 shows the recurrence periods based on the ignition mass of the first flash (filled circles) and obtained from the last shell flash (open squares) after several consecutive nova outbursts. The shell flashes were calculated by assuming mass loss in the expanded stages (Section 3). The calculations were continued until the repetition of the flashes became nearly a limit cycle. The calculations for longer recurrence times are very difficult because the shell flashes are very strong. For 


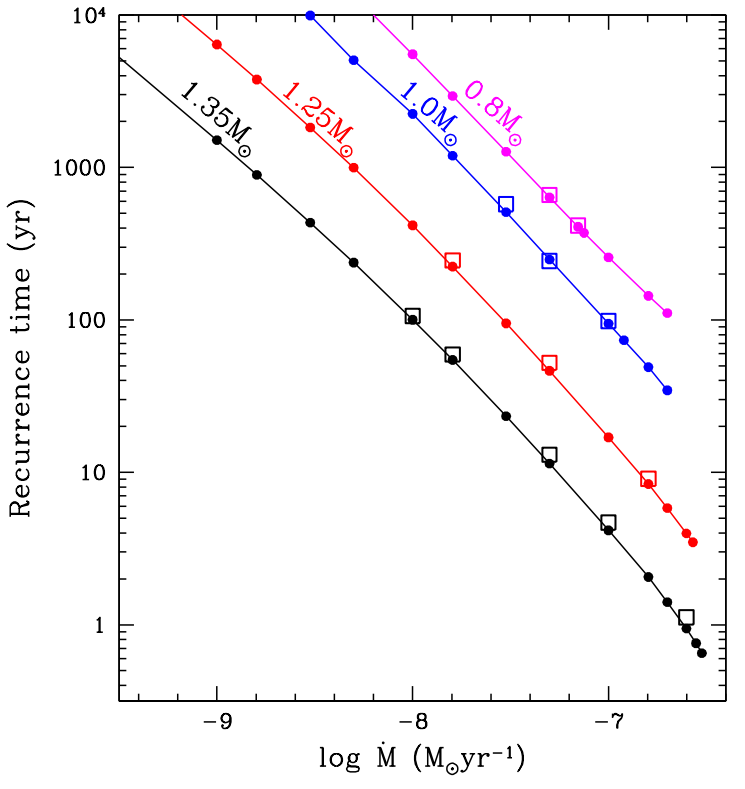

FIG. 9.- Recurrence period vs. mass accretion rate for 0.8, 1.0, 1.25, and $1.35 M_{\odot}$ WDs. Solid lines with small filled circles correspond to the recurrence time calculated from the envelope mass at the ignition of the first flash, as in Figure 6 and large open squares are the recurrence periods obtained by calculating many cycles of flashes until the repetition became nearly a limit cycle. See Section 4.4 for more details.

this reason, the recurrence periods obtained from multicycle calculations are limited to about a thousand years.

In the range where two types of recurrence periods are available, the periods agree with each other, as seen in Figure9 Actually, these two recurrence periods agree within
$10 \%$, suggesting that our estimates of the recurrence periods based on the first-cycle ignition mass and the minimum envelope mass given in Section 3.3 (Figure 6) are reasonable.

\section{CONCLUSIONS}

The main results are summarized as follows.

1. We proposed a physical mechanism that leads to a finite minimum recurrence period of novae.

2. We calculated the ignition masses for various WD masses and mass accretion rates. We determined that the shortest recurrence period of novae is about two months for a non-rotating $1.38 M_{\odot}$ WD with a mass accretion rate of $3.6 \times 10^{-7} M_{\odot} \mathrm{yr}^{-1}$.

3. A 1 yr recurrence period of a nova is possible only for very massive WDs $\left(M_{\mathrm{WD}} \gtrsim 1.3 M_{\odot}\right)$ and very high mass accretion rates, e.g., $\dot{M}=3.3 \times 10^{-7} M_{\odot} \mathrm{yr}^{-1}$ for a $1.31 M_{\odot} \mathrm{WD}, 2.4 \times$ $10^{-7} M_{\odot} \mathrm{yr}^{-1}$ for a $1.35 M_{\odot} \mathrm{WD}$, and $1.5 \times 10^{-7} M_{\odot} \mathrm{yr}^{-1}$ for a $1.38 M_{\odot}$ WD.

4. We revised our stability limit of hydrogen shell burning (Nomoto et al. 2007), which is useful for binary evolution calculations toward SNe Ia.

We thank the anonymous referee for useful comments that improved the manuscript. This research was supported in part by Grants-in-Aid for Scientific Research (22540254, 23224004, 23540262, 24540227, and 26400222) from the Japan Society for the Promotion of Science and by the WPI Initiative, MEXT, Japan.

\section{REFERENCES}

Canal, R., Isern, J., \& Labay, J. 1990, ARA\&A, 28183

Darnley, M. J., Williams, S. C., Bode, M. F., et al. 2014, A\&A, 563, L9

Eggleton, P., Faulkner, J., \& Flannery, P. 1973, A\&A, 23, 325

Fujimoto, M. K. 1982, ApJ, 257, 767

Hachisu, I., \& Kato, M. 2001, ApJ, 558, 323

Hachisu, I., \& Kato, M. 2003a, ApJ, 590, 445

Hachisu, I., \& Kato, M. 2003b, ApJ, 598, 527

Hachisu, I., \& Kato, M. 2006, ApJS, 167, 59

Hachisu, I., Kato, M., Nomoto, K., \& Umeda, H. 1999a, ApJ, 519, 314

Hachisu, I., Kato, M., \& Nomoto, K. 1999b, ApJ, 522, 487

Hachisu, I., Kato, M., \& Nomoto, K. 2010, ApJ, 724, L212

Han, Z., \& Podsiadlowski, Ph. 2004, MNRAS, 350, 1301

Henze, M., Ness, J.-U., Darnley, M., et al. 2014, A\&A, 563,L8

Hillebrandt, W., \& Niemeyer, J.C. 2000, ARA\&A, 38, 191

Iben, I. Jr. 1982, ApJ, 259, 244

Idan, I., Shaviv, N. J., \& Shaviv, G. 2013, MNRAS, 433, 2884

Iglesias, C. A., \& Rogers, F. J. 1996, ApJ, 464, 943

Kahabka, P., \& van den Heuvel, E. P. J. 1997, ARA\&A, 35, 69

Kato, M. 1980, Progress of Theoretical Physics, 64, 847

Kato, M. 1982, PASJ, 34, 173

Kato, M. 1983, PASJ, 35, 507

Kato, M., \& Hachisu, I. 1994, ApJ, 437, 802
Kato, M., \& Hachisu, I. 2012, BASI, 40, 393

Kovetz, A. 1998, ApJ, 495, 401

Kovetz, A., \& Prialnik, D. 1994, ApJ, 424, 319

Li, X.-D., \& van den Heuvel, E. P. J. 1997, A\&A, 322, L9

Neo, S., Miyaji, S., Nomoto, K., \& Sugimoto, D. 1977, PASJ, 29, 249

Nomoto, K. 1982, ApJ, 253, 798

Nomoto, K. 1984, ApJ, 277, 791

Nomoto, K., Saio, H., Kato, M. \& Hachisu, I. 2007, ApJ, 663, 1269

Nomoto, K., Thielemann, F.-K., \& Yokoi, K. 1984, ApJ, 286, 644

Pagnotta, A., \& Schaefer, B. E. 2014, ApJ, 788, 164

Paczyński, B. 1983, ApJ, 264, 282

Paczyński, B., \& Żytkow, A. N. 1978, ApJ, 222, 604

Prialnik, D., \& Kovetz, A. 1995, ApJ, 445, 789

Sala, G., \& Hernanz, M. 2005, A\&A, 439, 1061

Sienkiewicz, R. 1975, A\&A, 45, 411

Sienkiewicz, R. 1980, A\&A, 85, 295

Sion, E. M., Acierno, M. J., \& Tomczyk, S. 1979, ApJ, 230, 832

Starrfield, S., Iliadis, C., Timmes, F. X., et al. 2012, BASI, 40, 419

Tang, S., Bildsten, L., Wolf, W. M., et al. 2014, ApJ, 786: 61

van den Heuvel, E. P. J., Bhattacharya, D., Nomoto, K., \& Rappaport, S. 1992, A\&A, 262, 97

Wolf, W. M., Bildsten, L., Brooks, J., \& Paxton, B. 2013, ApJ, 777, 136 